\title{
Magnetic correlations around the Mott transition in the Kagomé lattice Hubbard model
}

\author{
T. Ohashi $\dagger^{1}$, S.-i. Suga ${ }^{1}$, N. Kawakami ${ }^{1}$, H. Tsunetsugu ${ }^{2}$ \\ ${ }^{1}$ Department of Applied Physics, Osaka University, Suita, Osaka 565-0871, Japan \\ ${ }^{2}$ Yukawa Institute for Theoretical Physics, Kyoto University, Kyoto 606-8502, Japan
}

\begin{abstract}
We study the magnetic properties around the Mott transition in the Kagomé lattice Hubbard model by the cellular dynamical mean field theory combined with quantum Monte Carlo simulations. By investigating the q-dependence of the susceptibility, we find a dramatic change of the dominant spin fluctuations around the Mott transition. The spin fluctuations in the insulating phase favor down to the lowest temperature a spatial spin configuration in which antiferromagnetic correlations are strong only in one chain direction but almost vanishing in the others.
\end{abstract}

PACS numbers: 71.30.+h 71.10.Fd 71.27.+a

Geometrical frustration is one of long-standing problems in spin systems. Recently, the frustration effects have attracted much attention also in itinerant electron systems. The observation of heavy fermion behavior in $\mathrm{LiV}_{2} \mathrm{O}_{4}$ [1, which has the pyrochlore lattice structure with a corner-sharing network of tetrahedra, has activated theoretical studies of electron correlations with geometrical frustration [2, 3, 4]. The discovery of superconductivity in the triangular lattice compound $\mathrm{Na}_{\mathrm{x}} \mathrm{CoO}_{2} \cdot \mathrm{yH}_{2} \mathrm{O}$ [5] and the $\beta$ pyrochlore osmate $\mathrm{KOs}_{2} \mathrm{O}_{6}$, 6] has further stimulated intensive studies of frustrated electron systems. Geometrical frustration has uncovered new aspects of the Mott metal-insulator transition. Among others, a novel quantum liquid ground state was suggested for the insulating phase of the triangular lattice [7], and this may be relevant for frustrated organic materials such as $\kappa-(\mathrm{ET})_{2} \mathrm{Cu}_{2}(\mathrm{CN})_{3}[8]$.

The Kagomé lattice (Fig. 1) is another prototype of frustrated systems showing many essential properties with the pyrochlore lattice. It is suggested that a correlated electron system on the Kagomé lattice can be an effective model of $\mathrm{Na}_{\mathrm{x}} \mathrm{CoO}_{2} \cdot \mathrm{yH}_{2} \mathrm{O}$ by properly considering anisotropic hopping matrix elements in the cobalt $3 d$ orbitals 9. The electron system on the Kagomé lattice in the metallic regime was studied recently by using the fluctuation exchange (FLEX) approximation [10] and quantum Monte Carlo (QMC) method [11], etc [12, 13]. In our recent paper [14], we have studied electron correlations in the Kagomé lattice Hubbard model, and found the first-order Mott transition at the Hubbard interaction $U / W \sim 1.37$ ( $W$ : band width).

$\dagger$ Present address: Condensed Matter Theory Laboratory, RIKEN, Wako, Saitama 351-0198, Japan e-mail: t-ohashi@riken.jp 
Magnetic correlations around the Mott transition in the Kagomé lattice Hubbard model 2
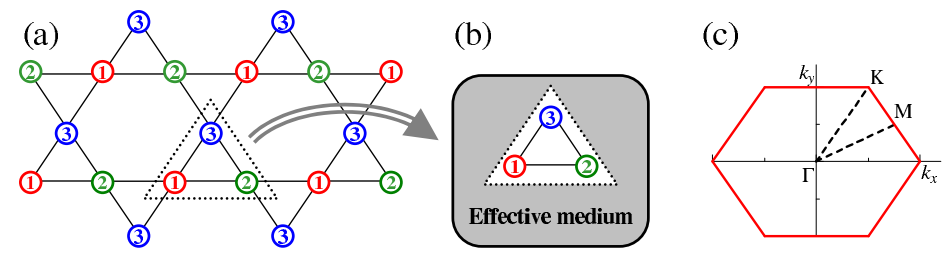

Figure 1. (a) Sketch of the Kagomé lattice and (b) the effective cluster model using three-site cluster CDMFT. (c) First Brillouin zone of the Kagomé lattice.

In this paper, we focus on the magnetic properties of the Kagomé lattice Hubbard model. By applying the cellular dynamical mean field theory (CDFMT) [15, we discuss the effects of geometrical frustration around the metal-insulator transition where frustration is stronger than in the weak coupling regime. We consider the standard Hubbard model with nearest-neighbor hopping $t>0$ on the Kagomé lattice,

$$
H=-t \sum_{\langle i, j\rangle, \sigma} c_{i \sigma}^{\dagger} c_{j \sigma}+U \sum_{i} n_{i \uparrow} n_{i \downarrow},
$$

with $n_{i \sigma}=c_{i \sigma}^{\dagger} c_{i \sigma}$, where $c_{i \sigma}^{\dagger}\left(c_{j \sigma}\right)$ creates (annihilates) an electron with spin $\sigma$ at the site $i$. In the following, we use the band width $W=6 t$ as the energy unit. The dynamical mean field theory (DMFT) [16] has given substantial theoretical progress in the field of the Mott transition but it does not incorporate spatially extended correlations. Therefore in order to take account of geometrical frustration, we use CDMFT, a cluster extension of DMFT [15, 17, 18, which has been successfully applied to frustrated systems such as the Hubbard model on the triangular lattice [19, 20, 21].

In CDMFT, the original lattice is regarded as a superlattice consisting of clusters, which is then mapped onto an effective cluster model via a standard DMFT procedure. As shown in Fig. 1, the Kagomé lattice Hubbard model is mapped onto a three-site cluster coupled to the self-consistently determined medium,

$$
S_{\text {eff }}=\int_{0}^{\beta} d \tau d \tau^{\prime} \sum_{\gamma, \delta, \sigma} c_{\gamma \sigma}^{\dagger}(\tau) \mathcal{G}_{\gamma \delta \sigma}^{-1}\left(\tau-\tau^{\prime}\right) c_{\delta \sigma}\left(\tau^{\prime}\right)+U \int_{0}^{\beta} d \tau \sum_{\gamma} n_{\gamma \uparrow}(\tau) n_{\gamma \downarrow}(\tau) .
$$

Given the Green's function for the effective medium, $\hat{\mathcal{G}}_{\sigma}$, we can compute the cluster Green's function $\hat{G}_{\sigma}$ and the cluster self-energy $\hat{\Sigma}_{\sigma}$ by solving the effective cluster model with QMC method [22]. Here, $\hat{\mathcal{G}}_{\sigma}, \hat{G}_{\sigma}$, and $\hat{\Sigma}_{\sigma}$ are described by $3 \times 3$ matrices. The effective medium $\hat{\mathcal{G}}_{\sigma}$ is then computed via the Dyson equation,

$$
\begin{aligned}
& \hat{\mathcal{G}}_{\sigma}^{-1}(\omega)=\left[\sum_{\mathbf{k}} \hat{g}_{\sigma}(\mathbf{k}: \omega)\right]^{-1}+\hat{\Sigma}_{\sigma}(\omega), \\
& \hat{g}_{\sigma}(\mathbf{k}: \omega)=\left[\omega+\mu-\hat{t}(\mathbf{k})-\hat{\Sigma}_{\sigma}(\omega)\right]^{-1},
\end{aligned}
$$

where $\mu$ is the chemical potential and $\hat{t}(\mathbf{k})$ is the Fourier-transformed hopping matrix for the superlattice,

$$
t_{\gamma \delta}(\mathbf{k})=\sum_{i, j} e^{-\mathbf{k} \cdot\left(\mathbf{r}_{i}-\mathbf{r}_{j}\right)} t_{\gamma \delta}(i, j) .
$$


Magnetic correlations around the Mott transition in the Kagomé lattice Hubbard model 3

Here the summation of $\mathbf{k}$ is taken over the reduced Brillouin zone of the superlattice (see Fig. 1(c)). After twenty iterations of this procedure, numerical convergence is reached. In each iteration, we typically use $10^{6}$ QMC sweeps and Trotter time slices $L=2 W / T$ to reach sufficient computational accuracy. Furthermore, we exploit an interpolation scheme based on a high-frequency expansion of the discrete imaginary-time Green's function obtained by QMC 23 in order to reduce time slice errors.

We now investigate the magnetic correlation around the Mott transition in the Kagomé lattice Hubbard model. We calculate the wavevector-dependence of the static susceptibility,

$$
\chi_{\gamma \delta}(\mathbf{q})=\int_{0}^{1 / T} d \tau \sum_{\mathbf{k}, \mathbf{k}^{\prime}}\left\langle c_{\mathbf{k} \gamma \uparrow}^{\dagger}(\tau) c_{\mathbf{k}+\mathbf{q} \gamma \downarrow}(\tau) c_{\mathbf{k}^{\prime}+\mathbf{q} \delta \downarrow}^{\dagger}(0) c_{\mathbf{k}^{\prime} \delta \uparrow}(0)\right\rangle,
$$

where $\gamma, \delta=1,2,3$ denote the site indices in the unit cell. We employ the standard procedure in DMFT to calculate $\chi_{\gamma \delta}(\mathbf{q})$ 16, which includes nearest-neighbor correlations as well as on-site correlations. In order to obtain $\chi_{\gamma \delta}(\mathbf{q})$, we first calculate the two-particle Green's function in the effective cluster model (2),

$$
\begin{aligned}
C_{\gamma \delta}\left(i \omega_{l}, i \omega_{m}\right) & =T \int_{0}^{\beta} \int_{0}^{\beta} \int_{0}^{\beta} \int_{0}^{\beta} d \tau_{1} d \tau_{2} d \tau_{3} d \tau_{4} \\
& \times e^{-i \omega_{l}\left(\tau_{1}-\tau_{2}\right)} e^{-i \omega_{m}\left(\tau_{3}-\tau_{4}\right)} C_{\gamma \delta}\left(\tau_{1}, \tau_{2}, \tau_{3}, \tau_{4}\right), \\
C_{\gamma \delta}\left(\tau_{1}, \tau_{2}, \tau_{3}, \tau_{4}\right) & =\left\langle\mathrm{T}_{\tau} c_{\gamma \uparrow}^{\dagger}\left(\tau_{1}\right) c_{\gamma \downarrow}\left(\tau_{2}\right) c_{\delta \downarrow}^{\dagger}\left(\tau_{3}\right) c_{\delta \uparrow}\left(\tau_{4}\right)\right\rangle,
\end{aligned}
$$

and extract the vertex function $\Gamma_{\gamma \delta}\left(i \omega_{l}, i \omega_{m}\right)$ via the Bethe-Salpeter equation,

$$
\hat{\Gamma}=\hat{C}^{0-1}-\hat{C}^{-1}
$$

where $C^{0}$ is the bare two-particle Green's function,

$$
C_{\gamma \delta}^{0}\left(i \omega_{l}\right)=-\frac{1}{T}\left[\sum_{\mathbf{k}} g_{\gamma \delta \downarrow}\left(\mathbf{k}: i \omega_{l}\right)\right]\left[\sum_{\mathbf{k}} g_{\delta \gamma \uparrow}\left(\mathbf{k}: i \omega_{l}\right)\right] .
$$

On the other hand, the bare q-dependent Green's function in the lattice system is calculated by

$$
C_{\gamma \delta}^{0}\left(\mathbf{q}: i \omega_{l}\right)=-\frac{1}{T} \sum_{\mathbf{k}} g_{\gamma \delta \downarrow}\left(\mathbf{k}+\mathbf{q}: i \omega_{l}\right) g_{\gamma \delta \uparrow}\left(\mathbf{k}: i \omega_{l}\right) .
$$

By using Eqs. (9) and (11), we can compute the lattice q-dependent Green's function,

$$
\hat{C}(\mathbf{q})=\left[\hat{C}^{0}(\mathbf{q})^{-1}-\hat{\Gamma}\right]^{-1} .
$$

Taking account of the phase factor, we finally obtain the q-dependent susceptibility,

$$
\chi_{\gamma \delta}(\mathbf{q})=T^{2} \sum_{l, m} C_{\gamma \delta}\left(\mathbf{q}: i \omega_{l}, i \omega_{m}\right) e^{-i \mathbf{q} \cdot\left(\mathbf{r}_{\gamma}-\mathbf{r}_{\delta}\right)} .
$$

It is convenient to introduce $\chi_{m}(\mathbf{q})$ for three normal modes $(m=1,2,3)$ by diagonalizing the $3 \times 3$ matrix $\chi_{\gamma \delta}(\mathbf{q})$. In the upper panels of Fig. 2, we show the three eigenmodes of the susceptibility at $T / W=1 / 30$ for several values of interaction strength $U / W$. In the noninteracting case, the largest eigenvalue of the susceptibility 
Magnetic correlations around the Mott transition in the Kagomé lattice Hubbard model 4

$U / W=0.0$

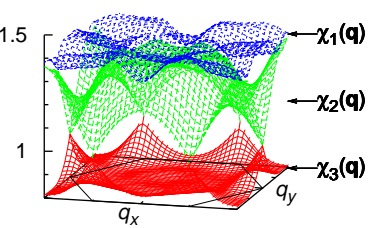

$\chi_{1}(\mathbf{q})$

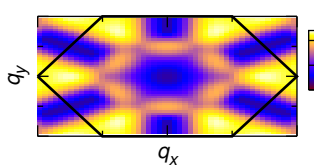

$U / W=0.6$
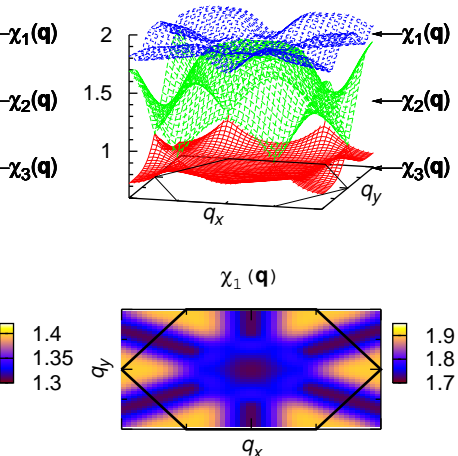

$\chi_{1}(\mathbf{q})$

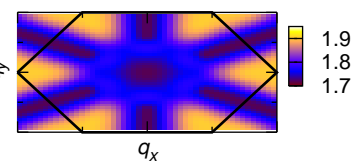

$U / W=1.1$

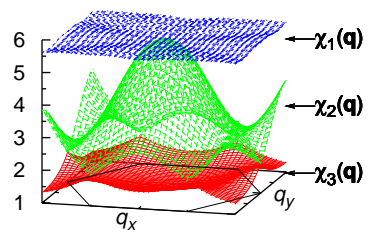

$\chi_{1}(\mathbf{q})$

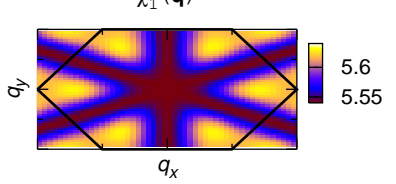

$U / W=1.4$

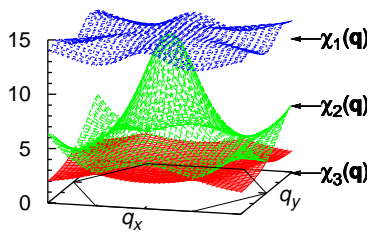

$\chi_{1}(\mathbf{q})$

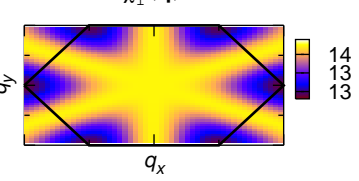

Figure 2. The wavevector dependence of the static susceptibility $\chi_{m}(\mathbf{q})$ for several values of $U / W$ at $T / W=1 / 30$. The three dimensional plots of $\chi_{m}(\mathbf{q})$ are shown in upper panels, from top to bottom $m=1,2,3$. The two dimensional plots in the lower panels show the dominant mode of the susceptibility $\chi_{1}(\mathbf{q})$ in upper panels. Hexagons in figures denotes the first Brillouin zone as shown Fig. 1 (c).
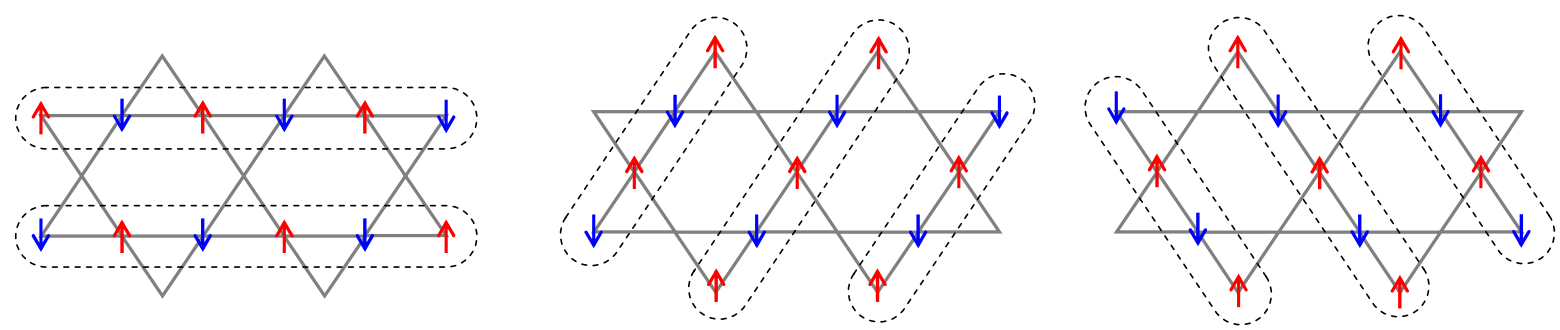

Figure 3. The enhanced spin correlations in the insulating phase $U / W=1.4$ at $T / W=1 / 30$.

$\chi_{1}(\mathbf{q})$ takes a maximum at six points in the Brillouin zone, the second largest one $\chi_{2}(\mathbf{q})$ has a maximum at $\mathbf{q}=(0,0)$, and the smallest one $\chi_{1}(\mathbf{q})$ takes maxima at the corners of the Brillouin zone. As $U / W$ increases, localized moments are formed and the susceptibility is enhanced as expected. In particular, the $\mathbf{q}=(0,0)$ peak of $\chi_{2}(\mathbf{q})$ becomes strongly enhanced, which is consistent with the previous QMC study 11. On the other hand, the dominant mode of the susceptibility $\chi_{1}(\mathbf{q})$ shows only weak q-dependence. As $U / W$ increases, $\chi_{1}(\mathbf{q})$ is enhanced not only at the six points above mentioned but also enhanced on the lines passing through $\Gamma$ and $M$ points, so that the q-dependence of the susceptibility gets suppressed and becomes much weaker at $U / W=1.1$ than in the noninteracting case. This behavior is consistent with the previous FLEX calculation in the weak coupling regime [10]. We confirm that the feature of the suppressed q-dependence of the dominant magnetic mode due to geometrical frustration persists up to fairly large- $U$ region.

We further find notable results in the insulating phase. The Mott metal-insulator transition occurs at $U / W \sim 1.37$ [14. As shown in the lower panels of Fig. 2, once the system enters the insulating phase, the $\mathbf{q}$-dependence of $\chi_{1}(\mathbf{q})$ dramatically 
Magnetic correlations around the Mott transition in the Kagomé lattice Hubbard model 5

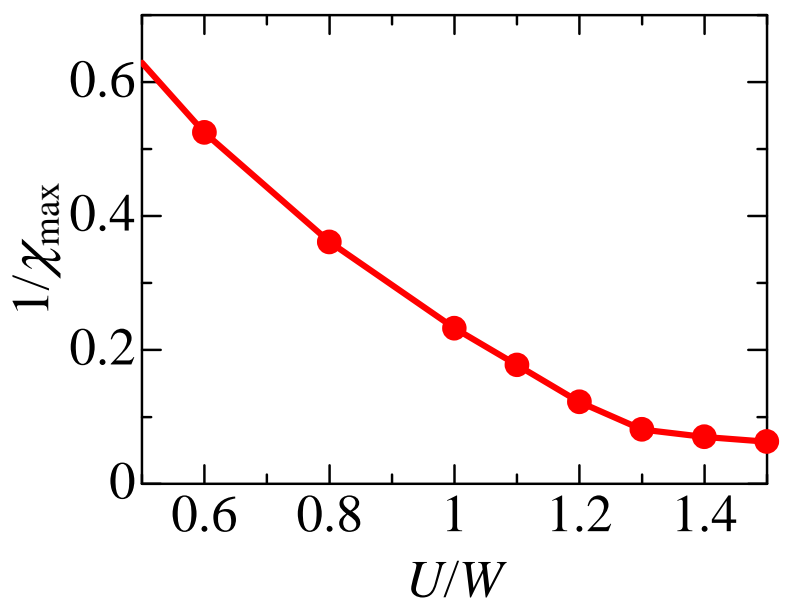

Figure 4. The inverse of the maximum susceptibility $1 / \chi_{\max }$ as a function of $U / W$ at $T / W=1 / 30$.

changes its character due to the enhancement of short range antiferromagnetic (AF) correlations [14]. At $U / W=1.4$, the susceptibility takes the maximum value along the three lines in $\mathbf{q}$ space instead of the six points in the weak coupling regime. Furthermore, by investigating the eigenvectors of $\chi_{1}(\mathbf{q})$, we find that two spins in the unit cell are antiferromagnetically coupled but the other spin is free. Therefore, at these temperatures, the enhanced spin fluctuations favor a spatial spin configuration in which one-dimensional (1D) AF-correlated spin chains are independently formed in three distinct directions. The three types of enhanced spin correlations are illustrated in Fig. 3. This spin correlation is one of the naturally expected spin correlation on the Kagomé lattice, because it stabilizes antiferromagnetic configurations in one direction, which is more stable than the naively expected spin configuration having a singlet pair and a free spin in each cluster. These $1 \mathrm{D}$ correlations in the finite- $T$ Mott insulating phase are different from the results for the Heisenberg model on the Kagomé lattice with the nearest-neighbor exchange obtained by both classical and semi-classical approximations 25. 24], but are similar to the $\mathbf{q}=0$ structure predicted for the classical Heisenberg model with a further neighbor exchange [24]. The essential difference from Ref. [24] is that there is almost no correlation between the different chains in our results for the Hubbard model. It remains an interesting problem to compare the q-dependence of the susceptibility in the large- $U$ and low- $T$ regime with the results for the Heisenberg model.

Finally, we show the maximum value of the susceptibility $\chi_{\max }$ as a function of $U / W$ in Fig. [. As $U / W$ increases towards the Mott transition point, $1 / \chi_{\max }$ decreases almost linearly as expected. When $U / W$ is further increased and the system enters the insulating phase, the spin correlation changes into the 1D one as discussed above, and then $\chi_{\max }$ saturates. Therefore, we find no evidence of the real instability to an 1D ordering in the present calculation. However, such enhanced spin fluctuations affect low-energy dynamics in the insulating phase [14]. 
In summary, we have studied the magnetic properties around the Mott transition in the Kagomé lattice Hubbard model by means of CDMFT combined with QMC. We have investigated the $\mathbf{q}$-dependence of the susceptibility, and obtained consistent results with the previous studies: the second-largest eigenmode of the susceptibility shows the strong $\mathbf{q}$-dependence, taking a maximum at $\mathbf{q}=(0,0)$, and the $\mathbf{q}$-dependence of the maximum eigenmode of the susceptibility becomes suppressed as $U$ increases in the metallic phase. We also find a dramatic change of the dominant spin fluctuations around the Mott transition. The spin fluctuations in the insulating phase favor a novel spatial spin configuration in which 1D AF-correlated spin chains are independently formed in three distinct directions. Although the spin liquid state or other nonmagnetic ordered states may be stabilized at zero temperature [26], the enhanced 1D spin correlations could emerge in the finite- $T$ Mott insulating phase.

The authors thank Y. Motome, A. Koga, and Y. Imai for valuable discussions. A part of numerical computations was done at the Supercomputer Center at ISSP, University of Tokyo and also at YITP, Kyoto university. This work was partly supported by Grant-in-Aid for Scientific Research (No. 16540313) and for Scientific Research on Priority Areas (No. 17071011 and 18043017) from MEXT.

\section{References}

[1] Kondo S, et al., 1997 Phys. Rev. Lett. 783729

[2] Lacroix C, 2001 Can. J. Phys. 791469.

[3] Tsunetsugu H, 2002 J. Phys. Soc. Jpn. 711844.

[4] Yamashita Y, and Ueda K, 2003 Phys. Rev. B 67195107.

[5] Takada K, et al., 2003 Nature (London) 42253.

[6] Yonezawa S, Muraoka Y, Matsushita Y, and Hiroi Z, 2004 J. Phys.: Cond. Mat. 16, L9.

[7] Kashima T, and Imada M, 2001 J. Phys. Soc. Jpn. 703052.

[8] Shimizu Y, Miyagawa K, Kanoda K, Maesato M, and Saito G, 2003 Phys. Rev. Lett. 91107001.

[9] Koshibae W, Maekawa W, 2003 Phys. Rev. Lett. 91257003.

[10] Imai Y, Kawakami N, and Tsunetsugu H, 2003 Phys. Rev. B 68195103.

[11] Bulut N, Koshibae W, and Maekawa S, 2005 Phys. Rev. Lett. 95037001.

[12] Läuchli A, and Poilblanc D, 2004 Phys. Rev. Lett. 92236404.

[13] Indergand M, Laeuchli A, Capponi S, and Sigrist M, cond-mat/0603401

[14] Ohashi T, Kawakami N, and Tsunetsugu H, 2006 Phys. Rev. Lett. 97066401.

[15] Kotliar G, Savrasov S Y, Pálsson G, and Biroli G, 2001 Phys. Rev. Lett. 87186401.

[16] Georges A, Kotliar G, Krauth W, and Rozenberg M J, 1996 Rev. Mod. Phys. 6813.

[17] Biroli G, Parcollet O, and Kotliar G, 2004 Phys. Rev. B 69205108.

[18] Maier T, Jarrell M, Pruschke T, and Hettler M H, 2005 Rev. Mod. Phys. 771027.

[19] Parcollet O, Biroli G, and Kotliar G, 2004 Phys. Rev. Lett. 92226402.

[20] Civelli M, Capone M, Kancharla S. S, Parcollet O, and Kotliar G, 2005 Phys. Rev. Lett. 95106402.

[21] Kyung B, and Tremblay A. -M. S, 2006, Phys. Rev. Lett. 97, 046402.

[22] Hirsch J E, and Fye R M, 1986 Phys. Rev. Lett. 562521.

[23] Oudovenko V S, and Kotliar G, 2002 Phys. Rev. B 65075102

[24] Harris A B, Kallin C, and Berlinsky A J, 1992 Phys. Rev. B 452899.

[25] Chubukov A, 1992 Phys. Rev. Lett. 69832.

[26] Misguich G, and Lhuillier C, Frustrated Spin Systems, edited by Diep H T (World Scientific, Singapore, 2004), and references therein. 